\title{
Téoros
}

Revue de recherche en tourisme

\section{Témoignages européens}

\section{Le tourisme en millieu autochtone}

\section{Michel Noël et Roselyne Boulard}

Volume 17, numéro 2, été 1998

L'industrie touristique autochtone

URI : https://id.erudit.org/iderudit/1072276ar

DOI : https://doi.org/10.7202/1072276ar

Aller au sommaire du numéro

Éditeur(s)

Université du Québec à Montréal

ISSN

0712-8657 (imprimé)

1923-2705 (numérique)

Découvrir la revue

Citer ce document

Noël, M. \& Boulard, R. (1998). Témoignages européens : le tourisme en milieu autochtone. Téoros, 17(2), 40-41. https://doi.org/10.7202/1072276ar d'utilisation que vous pouvez consulter en ligne.

https://apropos.erudit.org/fr/usagers/politique-dutilisation/ 


\section{TÉmOignAges EUROPÉENS}

\section{LE TOURISME EN MILIEU AUTOCHTONE}

\section{Propos recueillis par Michel Noël avec la collaboration de Roselyne Boulard}

La chose est bien connue : l'expérience possible de la culture et des milieux de vie autochtones exerce sur les Européens une fascination qui, souvent, influe même sur les motivations de leurs voyages au Québec. Le jeu en vaut-il la chandelle? En décembre 1994. quelques Européens se racontent, au lendemain de leur expérience touristique en milieu autochtone.

\section{PAROLES D'ÉDITH}

A vous tous, Thérèse, Roger, René, Marie-Louise, Mariette, Gayle, Louis, Benoît, Jacques, David, Nicole... tous ! Difficile de retrouver le quotidien. Mon esprit retoume souvent vers vous, vers tous ces échanges riches et émouvants. Il faudra du temps pour faire partager l'impartageable : les moments subtils qui laissent des traces indélébiles, un regard inconnu, un sourire les yeux plissés de malice, un geste venu de si loin, un geste si simple, si évident, l'odeur âpre du feu, de la forêt ; encore un regard rare de l'un d'entre vous, un regard d'avenir incertain et pourtant confiant.

Grâce à vous, ne plus se sentir * voyeur * mais $*$ faiseur $\%$, comme on entre dans la danse, d'abord timidement puis en oubliant les réticences ; prendre plaisir à quitter nos habituels vêtements, engoncés sous le poids de notre culture et de ses conventions.

Et quand tout devient urgent, se dire l'amitié et la solidarité, car rien n'est aussi essentiel.

Au-delà des impressions, merci à vous de $\mathrm{m}^{4}$ avoir ouvert la porte de vos possibles.
J'ai accueilli vos vies, nos rencontres, nos temps de partage, vos témoignages, avec humilité et émotion.

Vous avez su me faire percevoir votre résistance pour continuer à semer les graines reçues de vos ancêtres.

1995 : que cette semence ne soit ni privée de ses ailes, ni amputée de ses racines.

\section{PAROLES DE CATHERINE}

Une dizaine de jours, un voyage éclair et pourtant si intense, si plein de votre rencontre qu'il est bien difficile en quelques lignes de dire tout ce qu'il nous a offert. C'est vrai que Roselyne nous avait beaucoup expliqué le pays, les communautés, la forêt... Nous avons écouté, mais vous n'étiez pas là ; les lieux restaient diapositives et vos vies, histoires.

Aujourd'hui, sous vos visages je peux mettre des noms, au travers de la carte je peux tracer des itinéraires, derrière les contes et les légendes je vois le campement, le lac et le feu, dans le creux du tambour j'entends le sens de vos paroles. Vous nous avez parlé des jeunes et de leur mal de vivre, des anciens et de leur sagesse.

Vous nous avez raconté la vie ordinaire et initiés à ses rites.

Vous nous avez dit demain et cela si fort, que même dans le silence, sans qu'aucun mot ne soit prononcé, vos espoirs imprégnaient le vent, se mêlaient aux étincelles de nos soirées, enivraient les corps, donnaient un air de fête au temps partagé. Il y avait un énorme mouvement dans tout ce voyage, comme une lame de fond qui venait transcender le quotidien, nous éclaboussant au passage d'une multitude de questions sur le sens de l'identité recherchée.

Plus que par n'importe quelle démonstration, vous nous avez ouvert un champ entier à explorer qui est encore bien en friches chez nous.

\section{PAROLES DE JULIETTE}

Depuis dix ans, j'entendais parler des peuples autochtones, sans réussir à me faire une opinion personnelle. J'avais une connaissance théorique de votre histoire, de votre mode de vie, de vos problèmes sociaux, politiques et économiques ; et cela en restait là. Je m' intéressais davantage aux a étrangers $n$ que je pouvais rencontrer, soit chez eux dans leur pays ; soit en France.

Pour ce voyage, j'ai voulu être neutre, ouverte au maximum, c'est-à-dire sans a priori, sans préjugés, sans questions préalables. Je voulais écouter, regarder, sentir, oublier ma culture et mon histoire de 
femme occidentale, pour être totalement perméable à vous, à ce que vous alliez me faire comprendre, découvrir de vousmêmes (vous avez dû remarquer que j'etais la moins bavarde ; je me rattrape :)

Et j'ai été comblée au-delà de toute mesure. Je sais que je $\mathrm{n}^{\dagger}$ ai pas tout mémorisé : il y avait tant à assimiler en peu de temps. Il faudra d'autres voyages, d'autres rencontres, $d$ 'autres discussions, d'autres partages de la vie quotidienne.

Mais il y a déjà des lieux, des moments, des rencontres inoubliables. Je ne peux pas citer tous les noms, Roselyne me censurerait : * trop long *. Quelque chose reste, de très fort : RESPECT ! Pour vos différences, vos touts ; pour ce que vous êtes naturellement au jour le jour ; pour ce qui vous déchire mais que vous surmontez : la conciliation entre modernisme et tradition ; pour ce que vous tentez de mettre en place en préservant votre identité ou en aidant les jeunes à la découvrir et à en être fiers.

Et nous en France, si enrichis par cette rencontre avec vous, qu'allons-nous faire au plan individuel ? (Atoka agit déjà au nom de l'association.) Déjà rectifier les idées toutes faites sur vous - quand il y en a - ; donner le désir d'aller vers vous, avec le même esprit que le vôtre ; et puis pour le reste... c'est vous qui déciderez, non?

Merci

\section{PAROLES DE CHRISTOPHE}

Lignes ouvertes à des amis Atikamekw, Abénaquis, Algonquins

Oser quelques mots, quelques phrases pour tenter de dire, essayer de transmettre ce $\alpha$ entre $\%$, qui fit de ce voyage une extraordinaire aventure humaine :

Ce a entre $*$ les gens qui ne se voit pas, et qui est fait de regards curieux et complices,

Ce a entre $w$ les temps qui ne se comptent pas, et qui se rythme comme une respiration,

Ce \& entre * les mots qui ne suffisent plus, et qui se dit dans des silences partagés,
$\mathrm{Ce} *$ entre $\$$ les lignes que je ne saurai écrire, et que vous déchiffrerez, j'en suis sûr...

J'ai aimé ces rencontres avec des femmes, des hommes, des enfants, nos contemporains, voisins si lointains de ces milliers de kilomètres, et pourtant si proches, lors de ces temps d'échanges.

J'ai aimé ces rires partagés, ces temps d'humour complices à propos de tout, de rien, de mots, de situations.

J'ai aimé ce plaisir de la découverte d'une culture différente, d"une démarche spirituelle.

Jaimerais transmettre à ma fille, ce respect mutuel si riche de nos différences, cette relativité des choses, que la vie nous suggère, en de telles circonstances.

Merci, donc...

* Juste une manière de vivre, une manière d'être.

Mais il ne reste jamais rien de ce qui est vécu, quelques grains oxydés sur de la paraffine et des souvenirs...

Mais qui donne un peu de lumiêre les jours de pluic. $x$

Charlélie Couture

La ballade du mois d'août 1975

\section{PAROLES DE ROGER}

22 octobre -2 novembre 1994

Dix ans de partenariat, d'échanges, pour Roselyne et Atoka, avec les premières nations en terre québécoise : l'occasion d'un voyage exceptionnel, minutieusement préparé sans qu'il y paraisse, rondement mené sans pour autant qu'aucun de nous n'ait éprouvé la sensation de * toujours courir * que génère si facilement la soi-disant vie parisienne.

Ce voyage chez les Atikamekw, Abénaquis, partagé avec des Algonquins, m'a permis de retrouver, avec beaucoup d'émotion, sur leurs territoires, des hommes et des femmes, des amis - je le crois, je l'espère - rencontrés, accueillis en France lors des différentes manifestations culturelles organisées durant ces dix an-
Photo Socidte touristique Innu = M. G. Maillard, e. v. Saco.

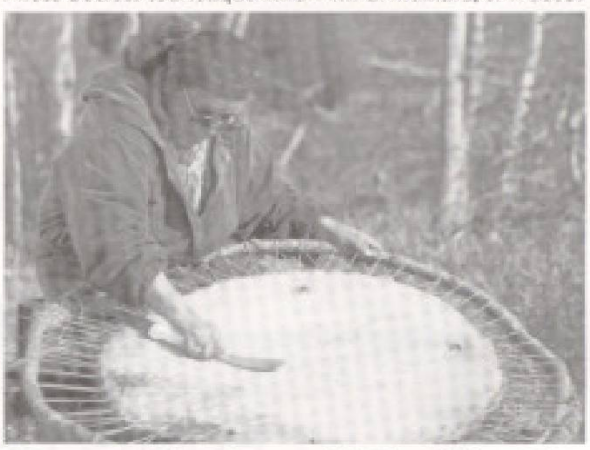

Démonstration d'une activité traditionnelle : le grattage de la peau de castor.

nées: Thérèse, Jacques à Coocoocache, Paul à Manawane, Geneviève manquée de si peu, Dominique mon vieux pirate nouvellement coiffé $\mathrm{d}^{+}$un chapeau curieusement indien...

Inutile de préciser que je pense très fort à Margot.

Et tous, tous ceux avec qui nous avons partagé ces temps forts, riches de sensations, de réflexions, d'échanges dont chacun retiendra ce qu'il voudra apporter dans son bagage : Marie-Louis, Davidet Marie, les joueurs de tambours, les familles et leurs bateaux. Claude, nos hôtes de La Tuque et d'ailleurs, Nicole, Rick et Donna, tous les autres, imprimés quelque part dans mon cour. dans un de ces recoins secrets où rien, jamais, ne disparaît.

De tout cela je retire une nouvelle fois le sentiment, somme toute banal, d'être un homme parmi les hommes, parmi tous les hommes, la certitude de n'avoir rien à imposer, a affirmer, comme celle de n'avoir rien à me laisser dicter, celle d'avoir plus à recevoir, peut-être, qu'à donner.

Nos croyances, nos cultures, nos histoires, nos différences, ne devraient être que sources d'échanges, de connaissances ćlargies, de savoirs cumulés, de respect, jamais de guerres, d'asservissement sournois, de paternalisme vulgaire.

Lieux communs que tout cela ? Pas si sûr !

En tout cas, Atoka, permettez-moi de souhaiter à tous et à chacun une belle année 1995 et d'espérer des retrouvailles futures, ici où là !

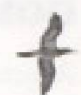

